\title{
A Novel routing protocol based on multipath routing for mobile adhoc networks
}

\author{
V.Sravani ${ }^{1}$, K.C.K.Naik ${ }^{2}$, Dr.Ch.Balaswamy ${ }^{3}$ \\ PG Student, Department of ECE, QIS College of Engineering \& Technology Ongole ${ }^{1}$ \\ Associate Professor, Department of ECE, QIS College of Engineering \& Technology Ongole ${ }^{2}$ \\ Professor \& HOD, Department of ECE, QIS College of Engineering \& Technology Ongole ${ }^{3}$
}

\begin{abstract}
Ad-hoc networks consist of a set of mobile nodes with a restricted power supply resources that can communicate with each other without any established infrastructure or centralized administration.In modern days communication plays a very important role. By considering the wireless communication networks Adhoc networks plays dominant role. The main problem of Adhoc network is route failure. To improve the life time of network different routing protocols are consider. In present routing protocols of ad hoc networks, routing is an act of moving information from a source to destination in an internetwork. Route is selected in the route discovery phase until all the packets are sent out. Due to the continuous flow of packets in a selected route leads to the route failure. In order to reduce this problem a novel routing protocol based on multipath considering the percentage of the optimum value is proposed. In this paper, the analysis of the performance of different routing protocols like DSR, MBCR and MMBCR to get maximum optimum value using Network Simulator Software is carried out.
\end{abstract}

Keywords: Ad hoc network, Route discovery phase, optimum value.

\section{INTRODUCTION}

Communication is activity of conveying information from source to destination. The proper communication should be maintain with the help of communication channel, the communication channel classified into two categories like wired communication channel and wireless communication channel. In present technologies improve Bandwidth capability, higher frequency signals and consideration of the losses the wireless communication channel is efficient than the wired communication channel. To maintain proper communication good communication network is needed. In communication process the network is called heart of communication system because in human body the heart is doing work circulation of blood to different organs as usually in communication process network can do passing the information from source to destination. Considering the different wireless communication networks like Infrared network, Bluetooth network, Wi-fi (wireless fidelity), Wimax (Wireless microwave access), Wireless sensor networks,Adhoc networks(Infrastructure less network-Advanced Developers Hands on Conference). We are working in the field of Mobile Adhoc networksLifetime enhancement by considering different protocols like DSR (Dynamic Source Routing Protocol), MBCR (Minimum Battery Cost Routing), MMBCR (Minimum Maximum Battery Cost Routing), MMBCR based on multipath routing. The communication between the nodes in a packet data network must be defined to ensure correct interpretation of the packets by receiving intermediate and end systems. Packet exchange between the nodes is called protocols. The routing involves two things:Firstly determining optimal routing paths, secondly transferring the information groups (called packets) through an internet work.
Routing protocols use several metrics to calculate the best path for routing the packets to its destination.Unsurprisingly, designing good protocols with few packets collision will reduce power consumption. At the network layer, the routing protocols can be designed such that there is an increase in the network life time by distributes the forwarding load over multiple different paths. A group of mobile devices called as nodes, without any centralized network, communicates with each other over multi-hop links is called as an Ad-hoc Network (MANET). A MANET is a collection of self organized mobile users which are free to act independently that communicate over relatively bandwidth constrained wireless links. Since the nodes are mobile, the network topology may change quickly and cannot be predicted over time. Figure 1.1 shows the flowchart for working of general ad-hoc network.

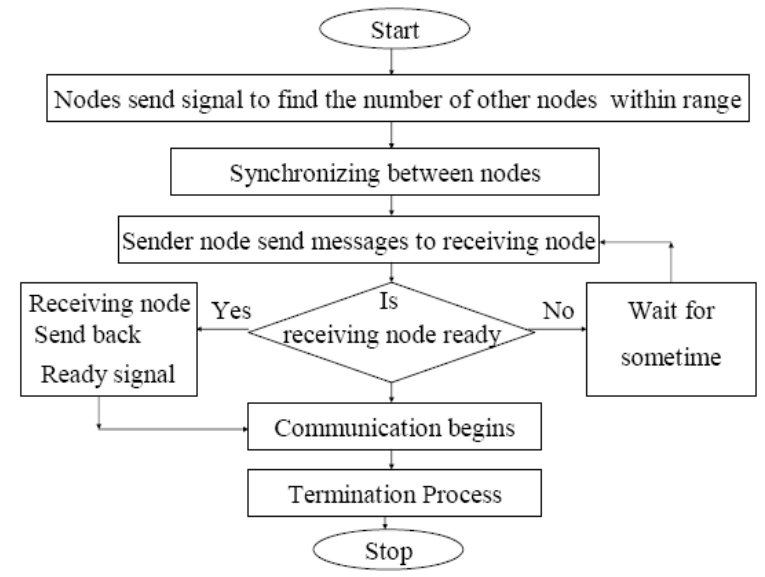

Figure 1.1 Working of general ad-hoc network 


\section{CLASSIFICATION OF ROUTING PROTOCOLS:}

Routing protocols for ad-hoc networks can generally be dividedinto three types. (i) Proactive Routing also known as table drivenrouting protocols (ii)Reactive routing also knows ason-demand routing protocols (iii) Hybrid routing protocols.

Each and every node has limited life spam. To maximize the life time of nodes in a network, the energy consumption rate of each node must be evenly distributed. Section II describes the theoretical analysis, Section III analyses the existing energy efficient routing protocols, and Section IV presents the proposed mechanisms to increase the network lifetime. Section V describes about the experimental results and lastly section VI gives the conclusion.

\section{II.THEORITICAL ANALYSIS}

Performance analysis between routing protocols for mobile ad-hoc networks we are considering different routing protocols like DSR-Dynamic Source Routing, MBCR-Minimum Battery Cost Routing, MMBCRMinimum Maximum Battery Cost Routing.

The Dynamic Source Routing (DSR) protocol is a simple and efficient routing protocol designed specifically for use in multi-hop wireless ad-hoc networks of mobile nodes. Using DSR, the network is completely self-organizing and self-configuring, requires no existing network infrastructure or administration. Network nodes co-operate to forward packets for each other to allow communication over multiple "hops" between nodes which are out of wireless transmission range from one another. As nodes in the network move about or join or leave the network, all routing is automatically determined and maintained by the DSR routing protocol.

Since the number or sequence of intermediate nodes needed to reach any destination may change at any time, the resulting network topology may be quite rich and rapidly changing. In DSR protocol overheads are very low and able to react very quickly to changes in the network. The DSR protocol provides highly reactive service in order to help ensure successful delivery of data packets in spite of node movement or other changes in network conditions.

The DSR protocol is composed of two main mechanisms that work together to allow the discovery and maintenance of source routes in an ad-hoc network:

Route Discovery: It is the mechanism by which a node S wishing to send a packet to a destination node $\mathrm{D}$ obtains a source route to D. Route Discovery is used only when $\mathrm{S}$ attempts to send a packet to D and does not already know a route to $\mathrm{D}$.

Route Maintenance: It is the mechanism by which node S is able to detect, while using a source route to $\mathrm{D}$, if the network topology has changed such that it can no longer use its route to D because a link along the route no longer works. When Route Maintenance indicates a source route is broken, $\mathrm{S}$ can attempt to use any other route it happens to know to $\mathrm{D}$, or it can invoke Route Discovery again to find a new route for subsequent packets to D. Route Maintenance for this route is used only when $S$ is actually sending packets to $\mathrm{D}$.

In DSR, Route Discovery and Route Maintenance each operate entirely "on demand". In particular, unlike other protocols, DSR requires no periodic packets of any kind. For example, DSR does not use any periodic routing advertisement, link status sensing, or neighbor detection packets. This entirely on-demand behavior and lack of periodic activity allows the number of overhead packets caused by DSR to scale all the way down to zero, when all nodes are approximately stationary with respect to each other and all routes needed for current communication have already been discovered. As nodes begin to move more or as communication patterns change, the routing packet overhead of DSR automatically scales to only what is needed to track the routes currently in use.

In response to a single Route Discovery, a node may learn and cache multiple routes to any destination. This support for multiple routes allows the reaction to routing changes to be much more rapid, since a node with multiple routes to a destination can try another cached route if the one it has been using should fail. This caching of multiple routes also avoids the overhead of needing to perform a new Route Discovery each time a route in use breaks. The sender of a packet selects and controls the route used for its own packets, which, together will support for multiple routes.

\subsection{The minimum battery cost routing (MBCR):}

This protocol was proposed in which use remaining battery capacity of each host as a metric to describe the lifetime of each host.

$$
f_{i}\left(c_{i}^{t}\right)=\frac{1}{c_{i}^{t}}
$$

Where, $f_{i}\left(c_{i}^{t}\right)$ is a battery cost function of a host $n_{\mathrm{i}}$. Now, suppose a node's willingness to forward packets is a function of its remaining battery capacity. The less capacity it has, the more reluctant it is. As the battery capacitydecreases, the value of cost function for node $n_{i}$ will increase. The battery cost $R_{j}$ for route $i$, consisting of D nodes, is

$$
\boldsymbol{R}_{j}=\sum_{i=0}^{D_{j}^{-1}} f_{i}\left(c_{i}^{t}\right)
$$

Therefore, to find a route with the maximum remaining battery capacity, we should select a route $i$ that has the minimum battery cost. 


$$
\boldsymbol{R}_{j}=\min \left\{\boldsymbol{R}_{j} \mid j \in A\right\}
$$

Where, $\mathrm{A}$ is the set containing all possible routes.

Advantage of MBCR: In MTPR, if the minimum total transmission power routes are via a specific host, the battery of this host will be exhausted quickly, and this host will die of battery exhaustion soon. Therefore, the remaining battery capacity of each host is a more accurate metric to describe the lifetime of each host. But, in MBCR since battery capacity is directly incorporated into the routing protocol, this metric prevents hosts from being overused, thereby increasing their lifetime and the time until the network is partitioned. If all nodes have similar battery capacity, this metric will select a shorter-hop route.

Disadvantage of MBCR: Because only the summation of values of battery cost functions is considered, a route containing nodes with little remaining battery capacity may still be selected. For example, in Figure 3.1 there are two possible routes between the source and destination nodes. Although node 3 has much less battery capacity than other nodes, the overall battery cost for route 1 is less than route 2 . Therefore, route 1 will be selected, reducing the lifetime of node 3 , which is undesirable.

\subsection{The min-max battery cost routing (MMBCR):}

This protocol was proposed in at first, in each possible route from source to destination, the maximum battery cost will be selected from Equation (2.2.1). Among this set of maximum battery costs, the minimum battery cost will be selected according to Equation (2.2.2). The battery of each host will be used more fairly than in previous schemes.

Battery cost $\mathrm{R}_{\mathrm{j}}$ for route $\mathrm{j}$ is redefined as

$$
R_{j}=\max \underset{i \in \text { route }_{-j}}{f_{i}}\left(c_{i}^{t}\right) \ldots \ldots
$$

Similarly, the desired route i can be obtained from the equation

$$
\boldsymbol{R}_{j}=\min \left\{\boldsymbol{R}_{j} \mid j \in A\right\} \ldots .(2.2 .2)
$$

Advantage: Since this metric always tries to avoid the route with nodes having the least battery capacity among all nodes in all possible routes, the battery of each host will be used more fairly than in previous schemes.

Disadvantage: The disadvantage is that since the minimum total transmission power is not considered in MMBCR, the power consumption may be more to transmit user traffic from a source to a destination, which actually reduces the lifetime of all nodes.

In MMBCR (Min-Max Battery Cost Routing) we first find the node having minimum battery capacity in each node of the possible routes and select the route having the maximum value among the selected routes. That means the route having maximum life time is selected. But themain demerit of MMBCR is that it does not consider the transmission powers of the nodes. In MMBCR, the updated information is not considered for route selection. So, two mechanisms are proposed to overcome this disadvantage. The first is MMBCR-route reply, where the cost function is calculated in route reply phase instead of in route request phase for selecting the route.

And the other is MMBCR with multipath route discovery to get more updated information about the routes. In this method periodically the route discovery process is done. If there are any changes in the route, the route information is updated. Because of this method, different routes are used for transmission of data packets and periodic shifting between the routes which avoids the over usage of nodes and node exhaustion leading to the increase of the life time of the network

\subsection{Performance Metrics:}

The following performance metrics are evaluated:

Packet delivery ratio:The ratio of the data packets delivered to the destinations to those generated by the CBR sources. Received packets and sent packets number could be easily obtained from the first element of each line of the trace file.

Average end-to-end delay: This includes all possible delays caused by buffering during route discovery latency, queuing at the interface queue, retransmission delays at the MAC, and propagation and transfer times.

Packet delivery ratio $(\%)=($ received packets/sent packets)*100

For each packet with id (Ii) of trace level (AGT) and type $(c b r)$, we can calculate the send $(s)$ time $(\mathrm{t})$ and the receive $(r)$ time $(\mathrm{t})$ and average it.

Routing overhead: It is the ratio of the routing packets sent and the total packets sent. Each hop-wise transmission of a routing packet is counted as one transmission.

Calculation of the routing overhead:

Routing overhead $=$ routing packets sent $/$ total packets sent

\subsection{Experiment Environment:}

Hardware: Laptop: CPU Intel Celeron M processor 370, 256MB Memory.

Operating System: Red hat 4, Windows XP

Network Simulator: ns-2, version 2.30 with CMU MANET extension.

Graph generator: gnu plot 4.20 


\section{III.EXISTING ROUTING MECHANISMS}

\section{ROUTE SELECTION BY DSR, MBCR AND MMBCR}

\subsection{ROUTE SELECTION BY DSR}

Let us consider a 7-node network shown in figure 3.1the route discovery process is started at $38 \mathrm{sec}$ to find the route from node 1 to node 4 . Since DSR does not consider the energies of the nodes and it only considers the minimum hop, the route $0-5-4$ is selected and the data packets are moving from 0 to 5 and from 5 to 4 as shown in the figure 5.5. Even though, there is less energy in the node 5 shown by the red circle, the DSR does not consider it and it selected the route $0-5-4$ since it is the shortest route with minimum hop.

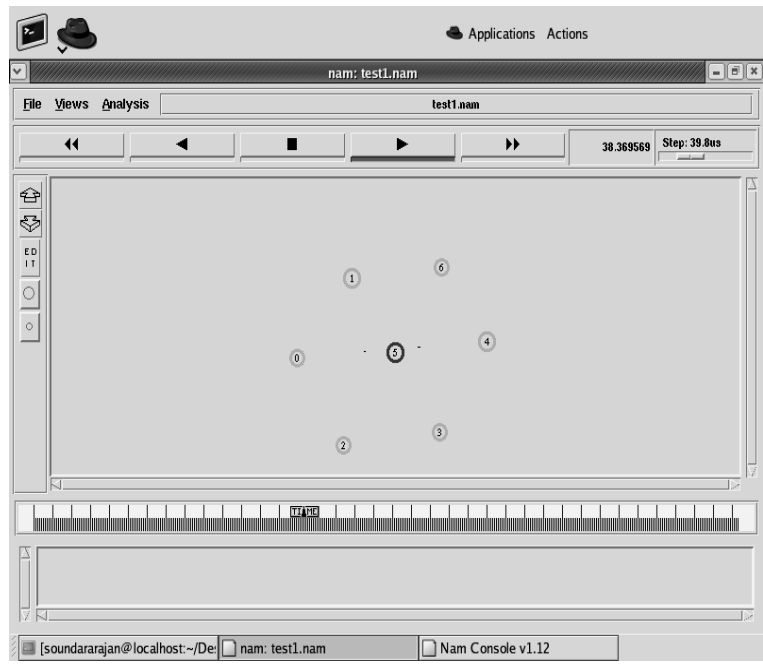

Fig. 3.1 A snapshot showing the route 0-5-4 is selected by DSR

This is the disadvantage of the DSR. The route does not exists longer time, since the energy of the node 5 exhausts quickly.

\subsection{ROUTE SELECTION BY MBCR}

Consider the same network as shown in fig 3.2, route discovery process starts at $38 \mathrm{sec}$ by MBCR protocol. At this time, according to the trace file generated in the NS2 simulator for the TCL script 1, the energy levels of all the nodes are Node $0-1.288755$, Node $1-1.237033$, Node 2 -1.239923 , Node $3-1.250182$, Node $4-1.290121$, Node $5-0.096358$ and Node $6-1.288979$. The corresponding cost functions are Node $0-0.776266$, Node $1-0.808429$, Node $2-0.806545$, Node $5-10.385178$, Node $6-$ 0.775945 , and Node $3-0.799978$. The total cost function along the route $0-2-3-4$ is 2.382789 , the total cost function along the route $0-5-4$ is 11.16144 , and the total cost function along the route $0-1-6-4$ is 2.360640 . Here, the route 0-1-6-4 has minimum total cost in the sense it has maximum battery capacity compared to the route $0-2-3-4$. Since MBCR selects the route with minimum total cost (with maximum battery capacity), the route $0-1-6-4$ is selected as shown in figure 3.2.

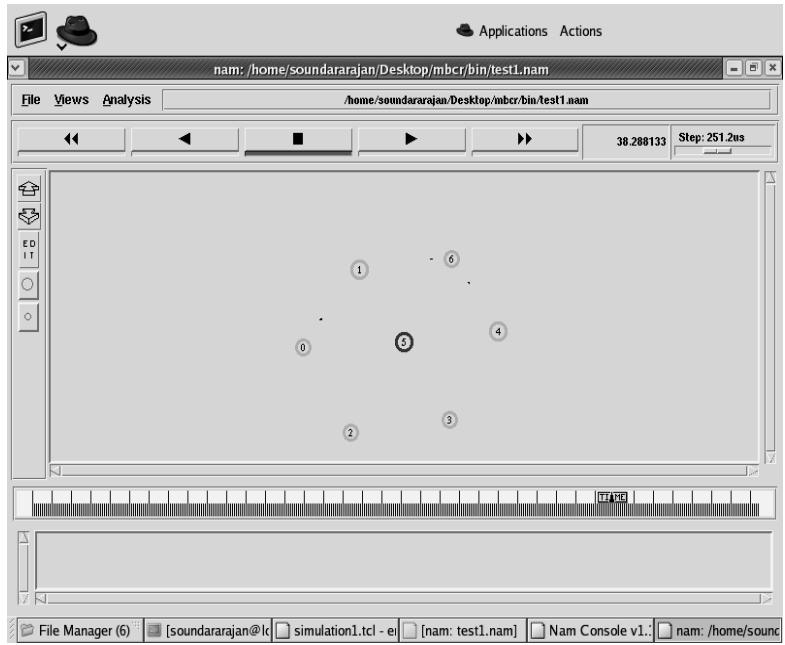

Fig. 3.2 A snapshot showing the route 0-1-6-4 is selected by MBCR

\subsection{ROUTE SELECTION BY MMBCR}

Consider the same network as shown in fig 3.3, route discovery process starts at $38 \mathrm{sec}$ by MMBCR protocol. At this time, according to the trace file generated in the NS2 simulator for the same TCL script 1 , the energy levels of all the nodes are Node $0-1.288755$, Node $1-1.237033$, Node 2 - 1.239923, Node 3 - 1.250182, Node 4 1.290121 , Node $5-0.096358$ and Node $6-1.288979$. The corresponding cost functions are Node $0-0.776266$, Node $1-0.808429$, Node $2-0.806545$, Node $5-10.385178$, Node $6-0.775945$, and Node $3-0.800080$. The MMBCR selects maximum battery cost (minimum battery capacity) in a route and stores. So, the maximum cost function in the route $0-2-3-4$ is 0.806545 , the maximum cost function in the route $0-5-4$ is 10.385178 , and the maximum cost function in the route 0-1-6-4 is 0.808429 . Since MMBCR selects the route with minimum cost function (maximum battery capacity) stored among all routes, the route $0-2-3-4$ is selected as shown in figure 3.3. The advantage of MMBCR is that it avoids the route which has a node with minimum battery capacity which leads to exhaust quickly.

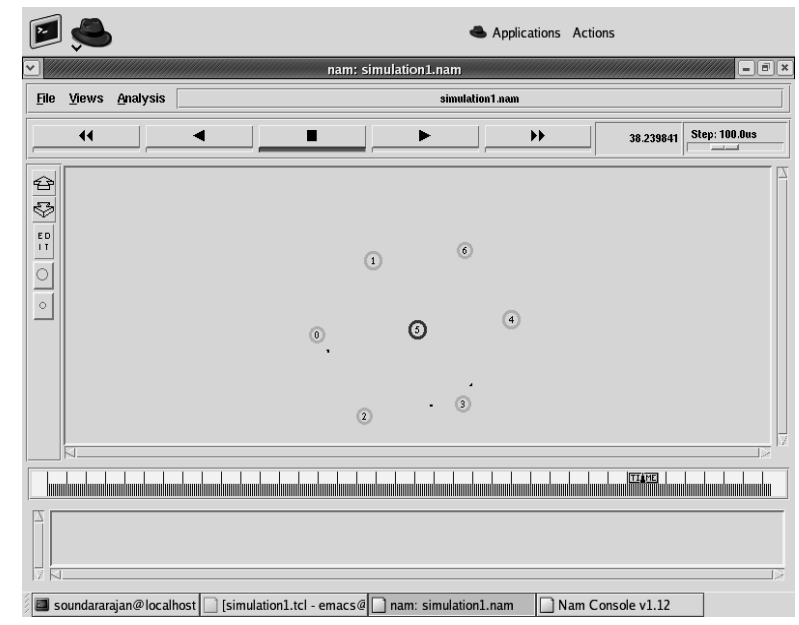

Fig. 3.3 A snapshot showing the route 0-2-3-4 is selected by MMBCR 


\section{IV.PROPOSED ROUTING MECHANISM}

In this mechanism we consider the routing protocol based on multipath.

In MMBCR the cost function is calculated and stored in route request packet header while going from source to destination. The decision of the route selection is made by the destination. Here the destination waits for some time to collect all the RREQ's.After making decision, it takes some time for RREP to reach the source. In between changes may occur in the energies of the network. The updated information is not considered in MMBCR for route selection. This can be overcome by calculating the cost function in RREP instead of in RREQ for route selection.Now, we are considering MMBCR-Route reply. Here the decision of route selection is made by the source node. The destination node simply replies to all the RREQs that reach it. Let us consider the source node which initializes the route request (RREQ).In between intermediate nodes plays a prominent role for the route selection. The intermediate node simply forwards the RREQ packet. And the destination node receives the RREQ packet to the corresponding route and responds immediately without any delay. The RREP and the intermediate node calculate their cost function and record the corresponding values in the RREP packet. And same method is followed and observed in the MMBCR of RREQ phase. The source node waits for some time and receives the entire RREP packet and selects the route with maximum life time and sends data packets through the route.The main advantage in this method is the updated information about the nodes is known and the best route is selected for the packets to transfer. One more advantage of this mechanism is that the source node receives all the possible routes for the destination. It stores the values in the cache memory for the future usage. But this process is not observed in MMBCR where the source receives only one route from the destination. Now, considering the MMBCR based on multipath, the problem observed in the existing MMBCR protocol is that once the route is selected in the route discovery phase. The selected route is used until all the data packets are sent out or until the selected route fails due to the exhaustion of node's battery. If any node in the selected route with less energy is observed then that node will certainly die out causing route failure and hence the total network lifetime is failed. And the main point observed is the nodes in the selected route suffer lot continuously due to the packets coming from other nodes. To reduce this problem a mechanism of multipath route discovery process is introduced. In this mechanism the route discovery process is initialized periodically to increase the network life time.

\section{V.EXPERIMENTAL RESULTS}

At first, the algorithms are developed to implement the efficient protocol routing and proposed mechanisms by using NS-2 Network Simulator in the environment of Red Hat Linux.

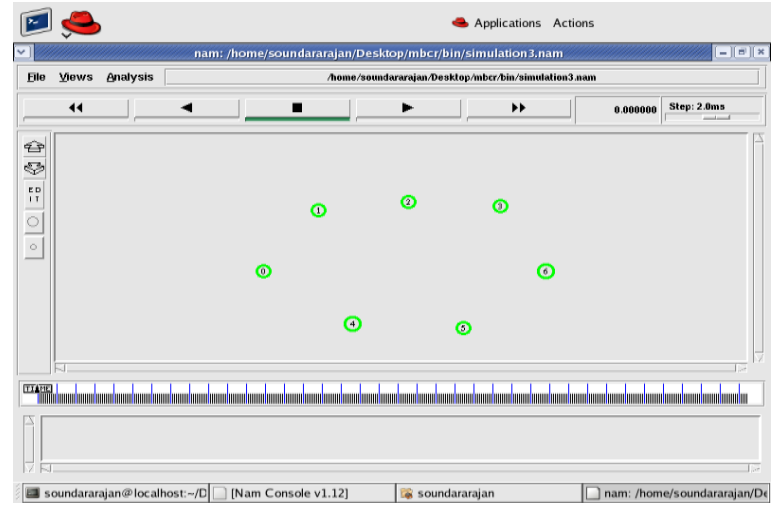

Fig 4.1A snapshot showing the route discovery process of nodes from 0 to 6

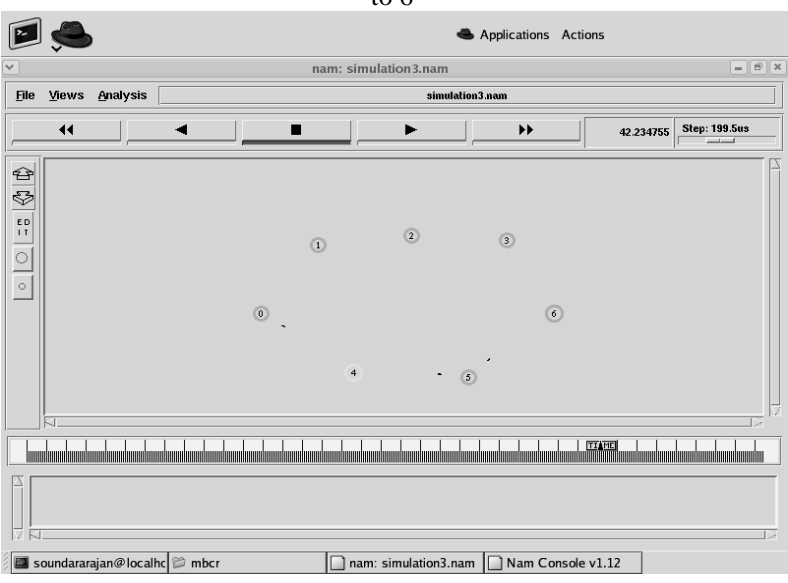

Fig 4.2 A snapshot showing the multipath process from 0-4-5-6 nodes

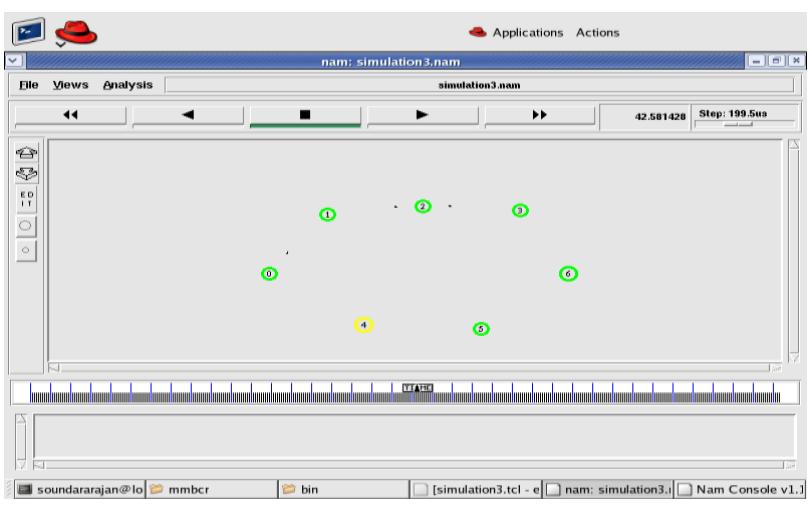

Fig 4.3. A snapshot showing the multipath process from nodes 0-1-2-3-6

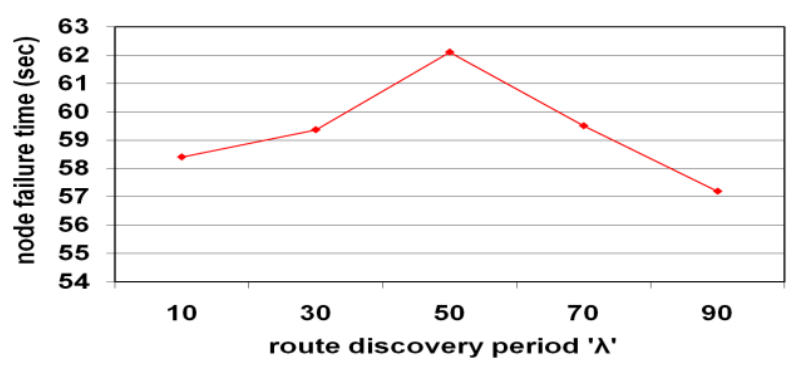

Fig 4.4. Node failure times for multipath route discovery MMBCR

In fig 4.1.we can observe the route discovery process is done first before the packets sent from the source to the destination. Here we have the nodes from 0-1-2-3-4-5-6, 
where 0 is the source node and 6 is the destination. The selection of the route is done by considering the cost functions of each nodes and energy levels. The cost functions of Node0-0.768620, Node1-0.983654, Node20.807313, Node3-0.931826, Node4-1.611672, and Node50.820880 .

In fig4.2.we can observe the periodic selection of the route. Here the route is selected which has higher battery capacity. The routing is observed from 0-4-5-6 which has the cost function of 1.611672.And also we can observe that in node4 battery capacity is decreased and due to the continuous flow of packets from source to destination. Due to this it may leads to exhaustion and finally to node failure and network failure. Since the MMBCR protocol avoids a node with the maximum battery cost, the route 0 4-5-6 is avoided and the next route is selected periodically without any delay.

In fig 4.3.we can observe the route selection is done from 0-1-2-3-6.MMBCR selects the route 0-1-2-3-6 which has higher battery capacity node. The cost function of this route is given as 0.983654 . In this the selection of the route is done same as in the route $0-4-5-6$.As the battery capacity of this route is high compared to the other route, this route is selected and will live longer in comparison with the other routes.

And finally from fig 4.in the proposed MMBCR with multipath routing protocol the route discovery period ' $\lambda$ ' is defined as the number of packets sent before the route discovery is reinitialized to find new route in which the battery energy is more in comparison with other possible routes. From the figure the graph between ' $\lambda$ ' and node failure time is taken.

By observing the results, it is noticed for the value $\lambda$ equal to 10 where the discovery is reinitialized for every 10 packets. As the value of $\lambda$ is increased to 50 node failure time also increases. This is because of the over usage of the single route to forward more number of packets which in turn decreases the battery capacity.

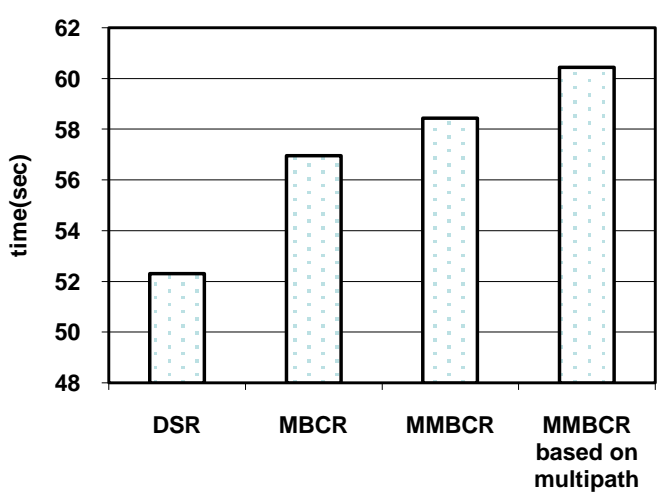

Fig. 4.5 Comparison of the route failure times of DSR, MBCR,MMBCR, MMBCR based on multipath routing

\section{VI.CONCLUSION}

In this paper, two mechanisms are proposed to increase the network life time. The first is MMBCR-Route reply, where the cost functions are calculated in route reply phase instead of in route request phase and the other is MMBCR based on multipath routing. In this if there is any updated information about the routes can be modified. And different route are used for the transmission of the data packets and periodic shifting between the routes is observed which avoids the over usage of nodes and node exhaustion leading to the increase of the network life time. The simulation results show that the proposed mechanism MMBCR based on multipath routing performs better in case of node failure time and the optimum period is investigated for MMBCR based on multipath routing to get higher node failure time.

\section{REFERENCES}

1) Dalia I.Elewely, MarwaF.Areed, HeshanA.Ali, "A Proposed routing scheme for power reduction in wireless networks", International Journal of Engineering \& Technology, doi:10.14419/ijet.v3i1.1111.

2) Mohammed Tarique, Kemal E. Tepe, and Mohammad Naserian, "Energy Saving Dynamic Source Routing for Ad Hoc Wireless Networks", Int. Proc. Of WIOPT, 2005.

3) S. Singh and C.S. Raghavendra, "PAMAS-Power Aware MultiAccess Protocol with signaling for Ad Hoc Networks", ACM Common. Rev., July 1998.

S. Singh, M. Woo, and C.S. Raghavendra, " Power Aware routing in Mobile Ad Hoc Networks, "Proc. Mobicom' 98, Dallas, TX, Oct 1998

5) C.K. Toh, "Maximum Battery Life Routing to support ubiquitous Mobile computing in Wireless Ad Hoc Networks", IEEE Communications Magazine, June 2001.

6) W. Cho and S.L. Kim, "A fully distributed routing algorithm for maximizing lifetime of a wireless ad hoc network, "4th Int. Workshop on Mobile and Wireless Communications Network, 2002, Sep2002, pp. 670-674.

7) The Network Simulator NS-2, http://www.isi.edu/nsnam/ns/ http://www.isi.edu/nsnam/ns/tutorial

\section{BIOGRAPHIES}

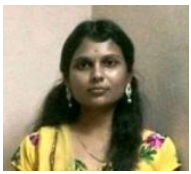

V. Sravani received the B.Tech Degree in ECE from QIS Institute of Technology, Ongole, A.P, in 2012. She is doing M.Tech in ECE Department with a specialization of DECS in QIS College of Engineering and Technology, Ongole. Her research interest is in Wireless Communication and Computer Networking.

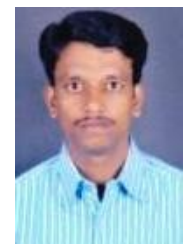

K.C.KullayappaNaik received the B.Tech and M.Tech Degrees in Electronics and Communication Engineering from MITS College, Madanapalle, A.P, in 2004 and 2007 respectively. He is doing his research work in Mobile Ad Hoc Networks. Now he is working as Associate Professor in ECE Department at QISCET, Ongole, Anandhra Pradesh, India.

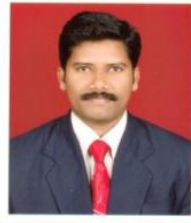

Dr.CH.BALASWAMY received the B.E degree in ECE from S.R.K.R. Engineering College, Bhimavaram in 1998. He received the M.Tech degree in ECE from Malnad College of Engineering, Hassan, India in 2001. He received his Ph.D from JNTU Anantapur in 2010. He has 13 years of experience in teaching for U.G and P.G students. He guided many B.Tech and M.Tech projects. He has published Seventeen International Journals and Six Research papers in National and International Conferences. His area of Interest is Mobile Adhoc Networks, Micro controllers and its applications. 